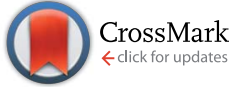

Cite this: RSC Adv., 2017, 7, 16444
Received 1st February 2017 Accepted 1st March 2017

DOI: 10.1039/c7ra01351e

rsc.li/rsc-advances

\title{
Influence of micelle formation on the adsorption capacity of a biosurfactant extracted from corn on dyed hair
}

\begin{abstract}
M. Rincón-Fontán, ${ }^{a}$ L. Rodríguez-López, ${ }^{a}$ X. Vecino, ${ }^{a b c}$ J. M. Cruz ${ }^{a}$ and A. B. Moldes ${ }^{\star a}$
Human hair contains fatty acids (palmitic, palmitoleic, oleic and stearic acid) that prevent hair dryness and avoid lower hair density of the scalp that can be caused when hair is dyed. These acids are also present in the composition of the amphoteric biosurfactant obtained from corn steep liquor, an agro-industrial stream generated by the corn wet-milling industry. This biosurfactant has a molecular weight of $1542 \mathrm{Da}$, with a similar mass spectrum to that of Fengycin, a biosurfactant produced by Bacillus subtilis strains. Few studies exist in the literature on the interaction of hair and biosurfactants, nor are there studies on the influence of micelle formation on biosurfactant adsorption capacity. Moreover, this is supposedly the first work in which a biosurfactant is applied to dyed hair. Different concentrations of biosurfactant were applied to dyed hair between $20-50{ }^{\circ} \mathrm{C}$ during 2-30 min of treatment. Theoretical models were obtained, which allowed the prediction of the amount of biosurfactant that can be entrapped by dyed hair. A maximum capacity of $10549 \mu \mathrm{g} \mathrm{g}^{-1}$ was achieved at $295 \mathrm{mg} \mathrm{L}^{-1}$ of biosurfactant, thus it could be observed that dyed hair mainly adsorbed the biosurfactant above its critical micellar concentration, at which point the biosurfactant is in micellar form. Furthermore, this treatment maintains the dyed hair structure in a good state.
\end{abstract}

\section{Introduction}

Recently, the presence of biosurfactants in the corn steep liquor (CSL) stream has been discovered, and the biosurfactants can be extracted by liquid-liquid extraction. ${ }^{1,2}$ The biosurfactant extract contained in this liquid stream was identified as a lipopeptide biosurfactant with a critical micellar concentration (CMC) of between 100-400 $\mathrm{mg} \mathrm{L}^{-1}$ (depending on the batch in the corn steep liquor) and has a capacity to reduce the surface tension of the medium in 32 units. The biosurfactant extract also contains antioxidants ${ }^{3}$ which could have a synergistic effect with other substances included in hair formulations or other cosmetic products for hair and cell protection, thus replacing synthetic surfactants.

Therefore, from an industrial point of view, the use of biosurfactants should play an important role in the cosmetic and pharmaceutical industries. ${ }^{4}$ There are a wide variety of studies on the function of biosurfactants as antimicrobial, ${ }^{5,6}$

${ }^{a}$ Chemical Engineering Department, School of Industrial Engineering - Centro de Investigación Tecnológico Industrial (MTI), University of Vigo, Campus As Lagoas-Marcosende, 36310 Vigo, Spain. E-mail: amoldes@uvigo.es; Tel: +34 986 812022

${ }^{b}$ Chemical Engineering Department, Escuela de Ingenieria de Barcelona Este (EEBE), Universitat Politècnica de Catalunya (UPC)-BarcelonaTECH, Campus DiagonalBesòs, 08930 Barcelona, Spain

${ }^{c}$ Barcelona Research Center for Multiscale Science and Engineering, Campus DiagonalBesòs, 08930 Barcelona, Spain anti-adhesive $\mathrm{e}^{7,8}$ and anti-tumoral agents ${ }^{9,10}$ with clear applications in the pharmaceutical industry. ${ }^{11-15}$ For instance, Rodrigues et al. ${ }^{15}$ have referenced the effect of a lipopeptide biosurfactant as an immunological adjuvant in rabbits and chickens. However, few studies on the use of biosurfactants in the cosmetic sector exist. ${ }^{4}$ Specifically, in terms of hair assays, almost no reports have been published on surfactants, with the reports by Ran and collaborators ${ }^{16}$ and Wagner et al., ${ }^{17}$ being studies focused on hair treatment with synthetic surfactants. In fact, regarding the application of biosurfactants on hair, in a previous work Rincón-Fontán and coworkers ${ }^{18}$ used a biosurfactant extract, obtained from CSL, on undamaged hair, and observed that samples of hair treated with biosurfactant had a more defined cuticle; it is necessary to remark that this biosurfactant was only evaluated at a specific concentration $\left(200 \mathrm{mg} \mathrm{L}^{-1}\right)$. Additionally, Morita et al. ${ }^{19}$ observed that mannosylerythritol lipids (MELs) had a positive effect on damaged hair, endowing it with better smoothness and flexibility.

Among all of the types of biosurfactants, it is estimated that the operational cost of producing lipopeptide biosurfactants, such as surfactin and iturin, is in the range of $10000 \$$ to $15000 \$$ per $\mathrm{g}^{20}$ assuming that the expense of the raw materials is the major outlay over the total production cost, and with the fermentation medium making up about $30 \%$ of the total payment. In the most favorable scenarios, biosurfactant prices are $20-30 \%$ superior to those of chemical surfactants, ${ }^{21}$ 
although the biosurfactant extracted from CSL could have similar costs to chemical surfactants because it is produced spontaneously. Up to now, research looking into industrial and agro-based waste and low-cost renewable substrates has led to significant biosurfactant cost reductions ${ }^{22-26}$ without paying attention to agro-industrial streams that possibly contain biosurfactants.

In this work, the biosurfactant extract obtained from CSL was applied, for the first time, to dyed hair, at concentrations below and above its CMC. The theoretical models obtained predict the best conditions to apply this biosurfactant onto dyed hair. In this way, this estimation should be very useful for the future production of shampoos or conditioner formulations. Furthermore, untreated dyed hair and hair treated with the biosurfactant have been characterized by scanning electron microscopy (SEM) analysis.

\section{Materials and methods}

\section{Biosurfactant extract from corn steep liquor}

The biosurfactant (BS) extract was obtained from the corn steep liquor (CSL) stream provided by Santa Cruz Biotechnology (Lot L1813). CSL, containing 50\% solids, was diluted in water up to $50 \mathrm{~g} \mathrm{~L}^{-1}$ and extracted with organic solvent (CSL solution :chloroform $1: 2 \mathrm{v} / \mathrm{v}$ ) at $56{ }^{\circ} \mathrm{C}$ for 60 min using the protocol established by Vecino and collaborators. ${ }^{2}$ Afterwards, the organic solvent was evaporated by vacuum distillation and the obtained biosurfactant extract was dissolved in water up to a concentration of $600 \mathrm{mg} \mathrm{\textrm {L } ^ { - 1 }}$.

The solution was passed through a cellulose filter paper $(45$ $\mu \mathrm{m}$, Tros Anoia, Spain) in order to eliminate insoluble solids. After that, $10 \mathrm{~mL}$ of the biosurfactant extract was taken and dried at $105{ }^{\circ} \mathrm{C}$ for $24 \mathrm{~h}$ in order to corroborate the concentration of the biosurfactant extract. All measurements were carried out in triplicate.

\section{Critical micellar concentration (CMC) of the biosurfactant extract}

The minimum concentration of biosurfactant needed to produce the maximum reduction in the surface tension (ST) of an aqueous solution is named the critical micellar concentration (CMC). Above the CMC, the surface tension of a biosurfactant solution is constant and specific for each biosurfactant. Below the CMC, the biosurfactant concentration is directly proportional to its capacity to reduce the ST of water. Thus, in order to establish this relationship, various solutions containing different biosurfactant extract concentrations were prepared by diluting the biosurfactant in water, and their surface tensions were measured using a tensiometer KRÜSS (K20 EasyDyne), using the Wilhelmy plate method. Measurements were carried out in triplicate, at room temperature, to increase the accuracy of the results. The reference $\mathrm{ST}$ value of demineralized water was $72 \mathrm{mN} \mathrm{m}^{-1}$.

\section{Physico-chemical characterization of the biosurfactant extract obtained from CSL}

Mass spectrometry sample preparation. The samples were analyzed by matrix-assisted laser desorption/ionization-time of flight mass spectrometry (MALDI-TOF) analysis. The drieddroplet sample preparation technique was used, applying $2 \mu \mathrm{L}$ of $\alpha$-cyano-4-hydroxycinnamic acid (CHCA) matrix solution (3 mg mL $\mathrm{m}^{-1}$ in ethanol/acetone/TFA $50: 50: 0.1 \mathrm{v} / \mathrm{v} / \mathrm{v}$ ) directly on a MTP AnchorChip ${ }^{\mathrm{TM}}$ 800/384 TF MALDI target (Bruker Daltonik, Bremen Germany) and, before drying the matrix solution, $2 \mu \mathrm{L}$ of concentrated biosurfactant extract was added and allowed to dry at room temperature. External mass calibration was performed using a calibration standard (Bruker Daltonik, Bremen Germany) for the range $m / z$ 700-3000 (9 mass calibration points). Afterwards, $0.5 \mathrm{~mL}$ of calibrant solution and CHCA matrix, previously mixed in an Eppendorf tube (1:2, v/v), was applied directly on the target and allowed to dry at room temperature.

MALDI-TOF-MS/MS analysis. Mass spectra were recorded using an Autoflex III smartbeam MALDI-TOF mass spectrometer (MALDI-TOF-MS) (Bruker Daltonik, Bremen, Germany), operating in the linear positive ion mode. Ions were formed upon irradiation by a smartbeam nitrogen laser (337 nm) using an accelerating potential of $20 \mathrm{kV}$ and a frequency of $200 \mathrm{~Hz}$. Each mass spectrum was produced by averaging 2500 laser shots, collected across the whole sample spot surface by rastering over the range $m / z 400-5000$. The laser irradiance was set to $65-70 \%$ (relative scale $0-100$ ) arbitrary units according to the corresponding threshold required for the applied matrix system. The low molecular ion gating was set to 390 daltons (Da) in order to remove the ions below this value arising from the matrix and their clusters or other unknown contaminants. All spectra were acquired and analysed using the flexControl 3.0 and flexAnalysis 3.0 software programs (Bruker Daltonik), respectively.

Following this, the samples were analysed similarly in order to obtain their mass-mass spectra by MALDI-TOF-MS/MS. The mass spectrometer was operated with positive polarity in the reflector mode. The peak corresponding to 1542 Da was fragmented using the Bruker LIFT method. It was fully accelerated to a kinetic energy of $27 \mathrm{kV}$ and a laser frequency of $200 \mathrm{~Hz}$. The laser irradiance was set at $20 \%$ to $40 \%$ of the maximum. A $1 \%$ (of parent ion mass) precursor ion selector (PCIS) window was used and 2000-2500 laser shots were usually accumulated for each spectrum. Similarly, the spectra were acquired and analysed using the same software programs as those used for the samples for the MS spectra.

Elemental analysis. C, N, H and S amounts were determined using a Fisons Carlo Erba EA-1108 CHNS-O elemental analyzer. Hence, the amount of $\mathrm{N}$ can be correlated with the protein content by multiplying it by a factor of $6.25{ }^{27}$

Fatty acid characterization. Following ISO-12966-3:2009 for fatty acid determination, aqueous samples were initially diluted in petroleum ether and dried with anhydrous sodium sulfate for $30 \mathrm{~min}$. Next, the drying agent was separated by paper filtration. The sample was mildly washed with petroleum ether, which was 
then removed by rotary evaporation (Buchi Rotavapor R-210, Switzerland).

After the pretreatment, $10 \mathrm{mg}$ of the sample was taken in a tube with $500 \mu \mathrm{L}$ of tert-butyl methyl ether (TBME). The sample was slightly heated when necessary. Following this, 250 $\mu \mathrm{L}$ of trimethylsulfonium hydroxide (TMSH) was added to the sample, and the sample tubes were stirred for $30 \mathrm{~s}$.

Separation of the fatty acid methyl esters (FAMEs) was carried out using a DB-WAX column $(30 \mathrm{~m} \times 0.25 \mathrm{~mm}$ i.d. $\times$ $0.25 \mu \mathrm{m}$ film thickness) with an oven temperature gradient of $50{ }^{\circ} \mathrm{C}$ for $2 \mathrm{~min}$, then heated from $50-220{ }^{\circ} \mathrm{C}$ at a rate of $4{ }^{\circ} \mathrm{C}$ $\min ^{-1}$ before being maintained for $15 \mathrm{~min}$. Helium was used as the carrier gas with a flow rate of $1 \mathrm{~mL} \mathrm{~min}^{-1}$ and the temperature of both the injector inlet and the transfer line of the detector was set at $250{ }^{\circ} \mathrm{C}$. A sample volume of $1 \mu \mathrm{L}$ was injected using a splitless mode.

Mass spectra were obtained using a mass selective detector under electron impact ionization at a voltage of $70 \mathrm{eV}$, and data were acquired over an $\mathrm{m} / \mathrm{z}$ range of 50-400. FAMEs were identified from the mass spectra library supplied with the GC-MS system and by comparison of the retention times and mass spectra of a FAME standard mix (Supelco 37 Component FAME Mix: $10 \mathrm{mg} \mathrm{mL} \mathrm{m}^{-1}$ of the FAME reference standard mix in methylene chloride, Sigma-Aldrich) injected under the same conditions. The software used to process the peak areas was MS Data Review (version 8.1).

Samples of dyed hair. Dyed blond human hair samples were kindly provided from a local hairdresser (from a 43 year old female). Before treatment, the hair was washed and rinsed out, until the surface tension of the demineralized water used during the cleaning process reached a value of $70-72 \mathrm{mN} \mathrm{m}^{-1}$. Afterwards, the dyed hair was dried for $24 \mathrm{~h}$, and kept in a paper envelope in order to conserve it. Its humidity was determined using a Denver IR-60 (UK) moisture analyzer, and was found to be $12.9 \pm 0.3 \%$. Furthermore, its length was measured in order to use samples that were as uniform as possible. Therefore, samples of dyed hair with lengths of about $35 \mathrm{~mm}$ were used during the experiments.

Adsorption experiments on dyed hair surfaces. Adsorption experiments were carried out in order to obtain theoretical models that could define the more favorable conditions for formulating and applying shampoos and conditioners, based on the biosurfactant extract obtained from CSL, onto dyed hair. An incomplete $3^{3}$ Box-Behnken factorial design was established in order to study the adsorption and interaction of the biosurfactant with dyed hair. This biosurfactant was contained in an aqueous solution, which was applied to dyed hair under different experimental conditions.

The temperature $\left(X_{1}\right)$, biosurfactant concentration $\left(X_{2}\right)$ and treatment time $\left(X_{3}\right)$ were fixed as independent variables, whereas the capacity of the dyed hair to entrap BS $\left(Y_{1}\right)$, and the percentage (\%) of BS removed from the aqueous solution $\left(Y_{2}\right)$ were defined as dependent variables. Table 1 shows the variation ranges established for the independent variables assayed, with their corresponding nomenclatures and units also shown. All of the variables were codified, with variation limits between -1 and 1 . Therefore, the variables are not influenced by their
Table 1 Independent and dependent variables used in the adsorption experiments

(a) Independent variables

\begin{tabular}{llll}
\hline Variable & Nomenclature & Units & Range of variation \\
\hline Temperature & $T$ & ${ }^{\circ} \mathrm{C}$ & $20-50$ \\
BS concentration & $\mathrm{BS}$ & $\mathrm{mg} \mathrm{L}^{-1}$ & $60-530$ \\
Time & $t$ & $\mathrm{~min}$ & $2-30$ \\
\hline
\end{tabular}

(b) Dimensionless, coded independent variables

\begin{tabular}{llll} 
Variable & Nomenclature & Definition & Range of variation \\
\hline Dimensionless $T$ & $X_{1}$ & $(T-35) / 15$ & $(-1,1)$ \\
Dimensionless BS & $X_{2}$ & $(\mathrm{BS}-295) / 235$ & $(-1,1)$ \\
Dimensionless $t$ & $X_{3}$ & $(t-16) / 14$ & $(-1,1)$ \\
\hline
\end{tabular}

(c) Dependent variables

\begin{tabular}{lll} 
Variable & Nomenclature & Units \\
\hline Adsorption capacity & $Y_{1}$ & $\mu \mathrm{g} \mathrm{g}^{-1}$ \\
Adsorption percentage & $Y_{2}$ & $\%$ \\
\hline
\end{tabular}

magnitudes, thus allowing the combination of the factors on a dimensionless scale.

A solid/liquid ratio of $1: 60(\mathrm{w} / \mathrm{v})$, in a batch operation system, at the different temperatures $\left(20-50{ }^{\circ} \mathrm{C}\right)$ established by the model in an orbital shaker at $150 \mathrm{rpm}$ was used for the adsorption experiments. At different intervals of time (2-30 min), stated by the incomplete $3^{3}$ Box-Behnken factorial design, samples of the BS solutions were obtained. The concentration of BS entrapped by dyed hair $\left(Y_{1}\right)$ and the adsorption percentages of BS $\left(Y_{2}\right)$ were quantified. Furthermore, Table 1 also includes the dependent variables studied in this work.

For comparative purposes, a solution containing $300 \mathrm{mg} \mathrm{L}^{-1}$ of hexadecyltrimethylammonium bromide (CTAB), which is a cationic synthetic surfactant used in cosmetic formulation, was added to the dyed hair in order to observe its effect on the hair structure.

Characterization of dyed human hair. The adsorption of the biosurfactant extract, from CSL, onto the dyed human hair surfaces was observed using a scanning electron microscope (SEM). First of all, the sample (a piece of dyed hair treated with the biosurfactant extract or treated with CTAB) was covered with a layer of gold (5-20 $\mu \mathrm{m})$ using an Emitech K550X (UK) sputter coater. Afterwards, SEM images were obtained using a Jeol JSM6700F FEG scanning electron microscope, operating at an acceleration voltage of $5.0 \mathrm{kV}$ for secondary-electron imaging (SEI).

Under the same conditions, an untreated dyed hair sample was used as a control.

Statistical analysis. The experimental data were analyzed by the response surface method with Statistica 7.0 software, by adjusting the obtained dependent experimental data to the quadratic function shown in eqn (1), where $Y$ is the dependent variable, $\beta$ denotes the regression coefficients (calculated from experimental data by multiple regressions using the least 
squares method), and $X$ denotes the fixed independent variables.

$Y=\beta_{0}+\beta_{1} X_{1}+\beta_{2} X_{2}+\beta_{3} X_{3}+\beta_{12} X_{1} X_{2}+\beta_{13} X_{1} X_{3}+\beta_{23} X_{2} X_{3}+$ $\beta_{11} X_{1}^{2}+\beta_{22} X_{2}^{2}+\beta_{33} X_{3}^{2}$

\section{Results and discussion}

\section{Biosurfactant extract characterization}

The relationship between the reduction in surface tension of water and the concentration of biosurfactant extract was evaluated in this work. This BS extract has a CMC of $75.41 \mathrm{mg} \mathrm{L}^{-1}$, with a capacity to reduce the surface tension of water in 32 units $\left(\mathrm{mN} \mathrm{m}^{-1}\right)$. Below the $\mathrm{CMC}$, a relationship between the biosurfactant concentration $(C)$ and the surface tension (ST) of water containing the BS can be established (Fig. 1).

This relationship is established in eqn (2).

$$
\mathrm{ST}=-0.44 C+80.14
$$

This biosurfactant extract was composed of $74.0 \% \mathrm{C}, 11.3 \%$ $\mathrm{H}$ and $1.5 \% \mathrm{~N}$, and the percentages of proteins and lipids were found to be $9.4 \%$ and $41.3 \%$, respectively.

Fig. 2 shows the MS and MS/MS spectra of the biosurfactant contained in the extract added to dyed hair. In the MS/MS spectrum, the peaks observed between 1506-1529 Da could correspond to the lipopeptide molecule. These fractions come from the fractionation of the peak situated at $1542 \mathrm{Da}$ in the MS spectrum, which conveys a molecular weight similar to that of Fengycin, a lipopeptide biosurfactant produced by the Bacillus subtilis strain composed of C16-C18 fatty acids. ${ }^{28,29}$ This is also in concordance with the fatty acids found in the biosurfactant extract obtained from CSL, and reported in previous works. ${ }^{1,3}$

Price and collaborators ${ }^{30}$ have also analyzed biosurfactants from 54 Bacillus strains and species from seven different geographical locations, using MALDI-TOF-MS/MS. They found that biosurfactants produced by Bacillus fell into three mass ranges: 850-950 m/z, which includes kurstakins, 1000-1100 m/z, which includes surfactins and iturins, and 1450-1550 m/z, which includes fengycins, polymyxins, and bacitracins.

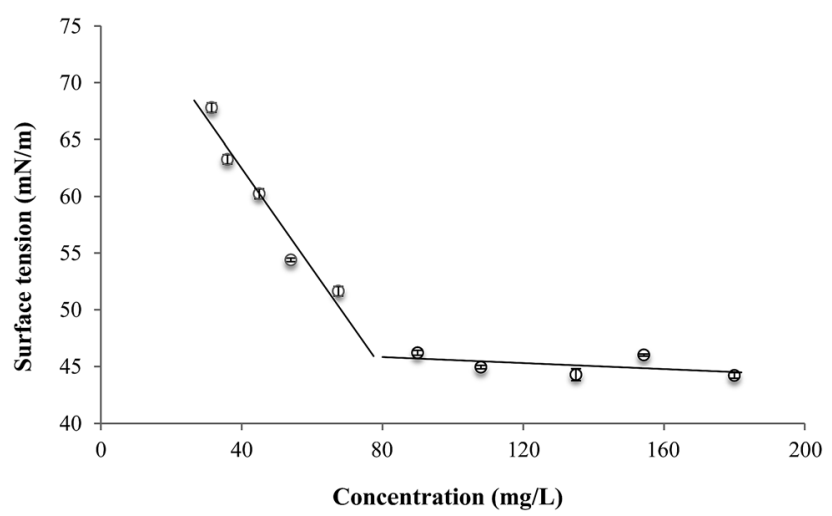

Fig. 1 Relationship between the surface tension of demineralized water and biosurfactant concentration.

\section{Adsorption experiments}

The experimental results obtained in this work revealed that dyed hair was able to entrap biosurfactant at concentrations above the CMC. A maximum adsorption capacity of about $10500 \mu \mathrm{g} \mathrm{g}^{-1}$ was obtained when working under intermediate conditions $\left(35^{\circ} \mathrm{C}\right.$, biosurfactant concentration equal to $295 \mathrm{mg}$ $\mathrm{L}^{-1}$ and a treatment time of $16 \mathrm{~min}$ ), whereas when the concentration of biosurfactant was $60 \mathrm{mg} \mathrm{L}^{-1}$ (below the CMC), no adsorption of biosurfactant by dyed hair was detected. This finding is in concordance with the data published by Ran et al. ${ }^{\mathbf{1 6}}$ working with dimethylpabamidopropyl laurdimonium tosylate (DDABDT), a quaternary ammonium surfactant. Table 2 shows the experimental conditions used, which are codified in order to avoid interferences due to their different scales, as well as the experimental results obtained in each case.

Until now, previous studies have obtained experimental capacities of adsorption with minor efficacy in comparison with this assay. For instance, the maximum experimental capacity obtained by Rincón-Fontán et al. ${ }^{18}$ on undamaged hair was 3679 $\mu \mathrm{g} \mathrm{g}^{-1}$, working with a concentration of biosurfactant of about $200 \mathrm{mg} \mathrm{L}^{-1}$. On the other hand, Ran et al. ${ }^{16}$ obtained capacities of $1500 \mu \mathrm{g} \mathrm{g}^{-1}$ and $4500 \mu \mathrm{g} \mathrm{g}^{-1}$ using DDABDT concentrations of 24.5 and $236 \mathrm{mg} \mathrm{L}^{-1}$, respectively.

Ran and collaborators, ${ }^{\mathbf{1 6}}$ working with DDABDT, observed that the surfactant concentration plays an important role in achieving the optimum treatment, and observed that the capacity of hair to entrap surface-active compounds increases above the CMC. These authors established that the association between hair and surfactants could be a monolayer or a bilayer structure, depending on the initial concentration of the surfactant. In the monolayer structure, surfactant molecules are adsorbed on hair surface fibers through electrostatic interactions, whereas in the bilayer structure, the second layer molecules may be piled up on top of the first layer with charged groups oriented outwards. Fig. 3 shows the possible adsorption mechanisms of the biosurfactant onto dyed hair. The results obtained in this work corroborate the existence of two different adsorption mechanisms as suggested previously by Ran and collaborators, ${ }^{16}$ consisting of a monolayer adsorption mechanism when the biosurfactant concentration is under the CMC (Fig. 3a) and a bilayer adsorption mechanism when the concentration is above the CMC (Fig. 3b).

Moreover, the results obtained by Ran et $a .^{16}$ are in good agreement with the data obtained in the present work, where it was observed that the concentration of biosurfactant is an important variable in the formulation of hair products, and it was noticed that concentrations lower than $60 \mathrm{mg} \mathrm{L}^{-1}$ repressed the adsorption of biosurfactant onto dyed hair, probably due to the vertical adsorption of biosurfactant onto hair as established by Ran et al. ${ }^{16}$

Otherwise, the results achieved in the current work are also in concordance with the ionic nature of BS, established in a previous work, ${ }^{31}$ where it was observed that this biosurfactant is amphoteric. The hair surface has a negative charge, so it adsorbs positively charged substances. In this case, the BS extracted from CSL has a positive and negative domain, therefore it can be entrapped by the hair surface and can also be used as a cleaning agent. Hair 

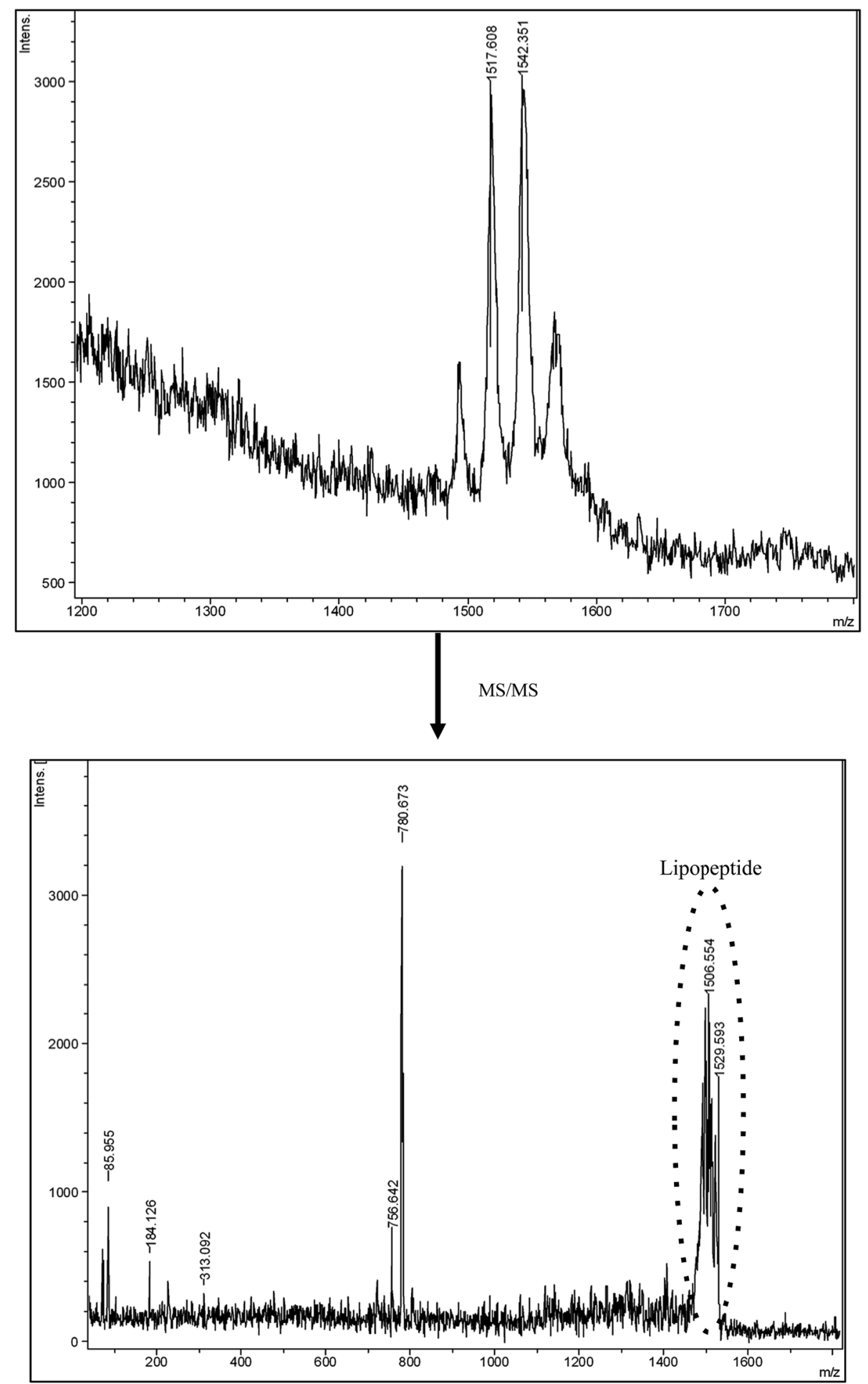

Fig. 2 Mass spectrum of the biosurfactant extract obtained from corn steep liquor and the mass-mass spectrum (MS/MS) of the peak obtained at $1542 \mathrm{~m} / \mathrm{z}$, respectively.

conditioners as well as hair sunscreens are formulated with positively charged surfactants and, in contrast, shampoos are formulated with negatively charged surface-active agents. ${ }^{32}$ Most shampoos are formulated with chemical surfactants that can induce, in many cases, hair protein loss, hair dryness, opacity and difficulty of handling. ${ }^{17,33,34}$
Establishment of theoretical models to predict the adsorption of biosurfactant from CSL onto dyed hair

After obtaining the experimental results, the efficacy of the method was corroborated by determining a theoretical model for the assay and studying the adsorption capacity of hair and 
Table 2 Operational conditions considered in this study (dimensionless temperature $X_{1}$, dimensionless BS concentration $X_{2}$ and dimensionless adsorption time $X_{3}$ ) and the experimental results achieved for the dependent variables $Y_{1}$, adsorption capacity and $Y_{2}$, adsorption percentage. Furthermore, the regression coefficients and their statistical significances for $Y_{1}$ and $Y_{2}$ were included (*significant coefficients, $p<0.05)$

\begin{tabular}{|c|c|c|c|c|c|}
\hline \multirow[b]{2}{*}{ Experiment } & \multicolumn{3}{|c|}{$\begin{array}{l}\text { Independent variables } \\
\text { (coded) }\end{array}$} & \multicolumn{2}{|c|}{ Dependent variables } \\
\hline & $X_{1}$ & $X_{2}$ & $X_{3}$ & $Y_{1}$ & $Y_{2}$ \\
\hline 1 & 0 & -1 & -1 & 967.05 & 27.97 \\
\hline 2 & 0 & 1 & -1 & 5065.18 & 17.42 \\
\hline 3 & 0 & -1 & 1 & 0.00 & 0.00 \\
\hline 4 & 0 & 1 & 1 & 155.24 & 0.53 \\
\hline 5 & -1 & -1 & 0 & 0.00 & 0.00 \\
\hline 6 & -1 & 1 & 0 & 8324.80 & 29.39 \\
\hline 7 & 1 & -1 & 0 & 0.00 & 0.00 \\
\hline 8 & 1 & 1 & 0 & 1178.98 & 4.05 \\
\hline 9 & -1 & 0 & -1 & 8693.68 & 46.14 \\
\hline 10 & -1 & 0 & 1 & 7432.26 & 44.63 \\
\hline 11 & 1 & 0 & -1 & 10273.25 & 60.34 \\
\hline 12 & 1 & 0 & 1 & 7072.94 & 48.62 \\
\hline 13 & 0 & 0 & 0 & 10549.07 & 53.75 \\
\hline 14 & 0 & 0 & 0 & 10341.33 & 53.84 \\
\hline 15 & 0 & 0 & 0 & 10464.42 & 54.48 \\
\hline
\end{tabular}

\begin{tabular}{lllll}
\hline \multirow{5}{*}{ Regression coefficients } & \multicolumn{4}{l}{ Statistical significance } \\
\cline { 2 - 5 } & $Y_{1}$ & $p_{Y_{1}}$ & $Y_{2}$ & $p_{Y_{2}}$ \\
\hline$\beta_{0}$ & 10451.61 & $0.00003^{*}$ & 54.02 & $0.00002^{*}$ \\
$\beta_{1}$ & -740.70 & $0.00248^{*}$ & -0.89 & $0.02401^{*}$ \\
$\beta_{11}$ & -627.25 & $0.00743^{*}$ & -3.61 & $0.00330^{*}$ \\
$\beta_{2}$ & 1719.64 & $0.00046^{*}$ & 2.93 & $0.00231^{*}$ \\
$\beta_{22}$ & -7448.41 & $0.00005^{*}$ & -42.06 & $0.00002^{*}$ \\
$\beta_{3}$ & -1292.34 & $0.00082^{*}$ & -7.26 & $0.00038^{*}$ \\
$\beta_{33}$ & -1456.33 & $0.00139^{*}$ & -0.49 & 0.14456 \\
$\beta_{12}$ & -1786.46 & $0.00085^{*}$ & -6.34 & $0.00099^{*}$ \\
$\beta_{13}$ & -484.72 & $0.01141^{*}$ & -2.55 & $0.00606^{*}$ \\
$\beta_{23}$ & -985.72 & $0.00280^{*}$ & 2.77 & $0.00516^{*}$ \\
& & & &
\end{tabular}

the adsorption percentage of biosurfactant onto dyed hair as dependent variables.

First of all, an incomplete $3^{3}$ Box-Behnken factorial design was stated, and theoretical equations, with all regression coefficients, for the adsorption capacity of hair (eqn (3)) and the adsorption percentage (eqn (4)) were obtained. Table 2 shows the coefficients obtained after statistical treatment.

$Y_{1}=10451.6-740.7 X_{1}+1719.6 X_{2}-1292.3 X_{3}-1786.4 X_{1} X_{2}-$ $484.7 X_{1} X_{3}-985.7 X_{2} X_{3}-627.3 X_{1}^{2}-7448.4 X_{2}^{2}-1456.3 X_{3}^{2}$

$Y_{2}=54.02-0.89 X_{1}+2.93 X_{2}-7.26 X_{3}-6.34 X_{1} X_{2}-2.55 X_{1} X_{3}+$

$2.77 X_{2} X_{3}-3.61 X_{1}^{2}-42.06 X_{2}^{2}-0.48 X_{3}^{2}$

On the other hand, for the hair adsorption capacity and adsorption percentage of biosurfactant onto dyed hair, it was observed that the biosurfactant concentration was by far the most influential variable, followed by treatment time, whereas the temperature was the least influential variable.

The variations of dyed hair adsorption capacity and the adsorption percentage in terms of the concentration of biosurfactant and time (most influential variables) were studied, fixing the temperature at its intermediate value (Fig. 4a and $\mathrm{b}$ respectively) established in the experimental design.

When treatment of dyed hair was carried out at a biosurfactant concentration of $60 \mathrm{mg} \mathrm{L}^{-1}$ (below the CMC), the model predicts that hair can adsorb until $1702 \mu \mathrm{g}$ of the biosurfactant is adsorbed per $\mathrm{g}$ of dyed hair, thus it was observed that the best capacity corresponds to the intermediate time stated (16 min). Regarding the adsorption percentage, the model predicts that hair can adsorb between $0-19 \%$, thus it was observed that the best results were obtained for the minor time stated $(2 \mathrm{~min})$. However, when the biosurfactant concentration was increased up to $295 \mathrm{mg} \mathrm{L}^{-1}$ (above 4 times the CMC) it was observed that a lower treatment time ( $2 \mathrm{~min}$ ) translated to the best dyed hair adsorption capacity, as well as the best adsorption percentage, with values ranging from 5850 to $10451 \mu \mathrm{g} \mathrm{g}^{-1}$ and $39-59 \%$, respectively. It is interesting to remark that dyed hair showed a higher adsorption capacity of biosurfactant in comparison with undamaged hair. Thus, in a previous study ${ }^{18}$ it was observed that undamaged hair in contact with the biosurfactant, extracted from CSL, at $200 \mathrm{mg}$ $\mathrm{L}^{-1}$ showed a capacity of about $3679 \mu \mathrm{g} \mathrm{g}^{-1}$, whereas in this work, the model predicts a capacity of about $10451 \mu \mathrm{g} \mathrm{g}{ }^{-1}$. It can be speculated that, in dyed hair, cuticles are more separated with a higher content of open pores, thus increasing the specific surface area. a)

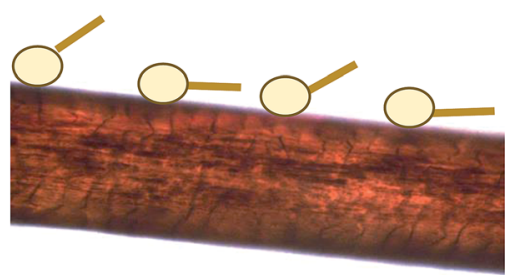

b)

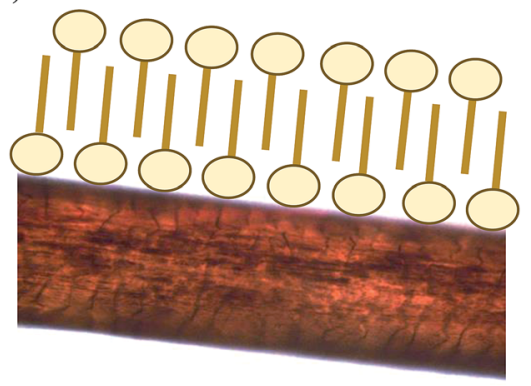

Fig. 3 Scheme of the behavior of the biosurfactant molecules in contact with dyed hair: monolayer (a) or bilayer (b), respectively. 
a)

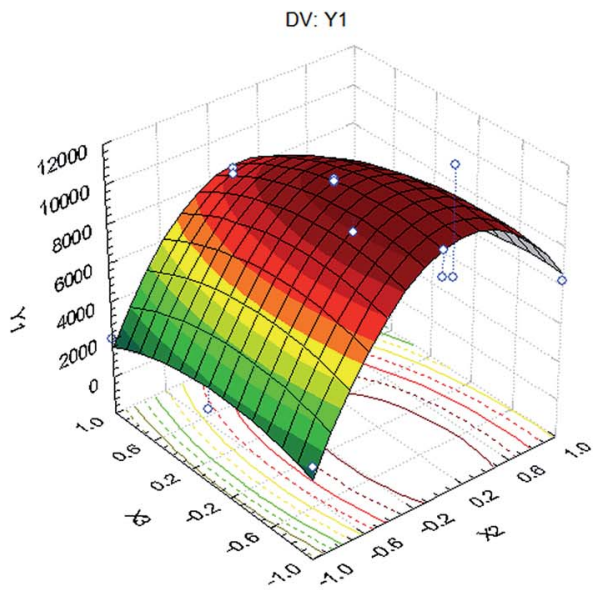

b)
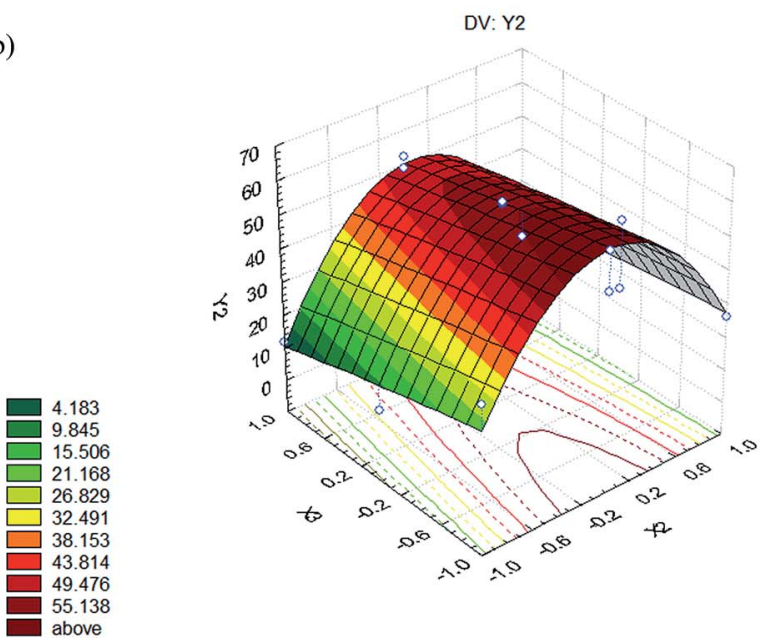

Fig. 4 Variation in the adsorption capacity of hair (a) and in the adsorption percentage of biosurfactant (b) respectively, depending on the variables $X_{2}$ (biosurfactant concentration), $X_{3}$ (treatment time) and $X_{1}$ (temperature) fixed at their intermediate value.

Finally, when the biosurfactant concentration was established as $530 \mathrm{mg} \mathrm{L}^{-1}$ (above 7 times the CMC), the best result for the adsorption capacity was obtained for the minor treatment time $(2 \mathrm{~min})$ and the lower temperature value $\left(20^{\circ} \mathrm{C}\right)$, with capacities of 988 to $6622 \mu \mathrm{g} \mathrm{g}^{-1}$, and adsorption percentages of $4-19 \%$.

In this way, it can be stated that the theoretical values of the adsorption capacities and percentages, obtained using eqn (3) and (4), respectively, are in concordance with those obtained in the experiments (Table 2).

Moreover, it was observed that the maximum capacity was obtained at a BS concentration of $295 \mathrm{mg} \mathrm{L}^{-1}$. This phenomenon could be explained as a consequence of the saturation of dyed hair. It seems that in the case of using more concentrated solutions of BS, the hair cannot adsorb it with the same efficacy than when using less concentrated solutions, all of them above the CMC. Furthermore, it is necessary to take into account that the biosurfactant extract, obtained from CSL, contains other substances like antioxidants or free fatty acids ${ }^{3}$ that can interfere in the adsorption of biosurfactant when it is concentrated at many times over its CMC.

\section{Morphology of the dyed hair surface before and after adsorption of the biosurfactant from CSL}

In order to understand the morphology of the hair surface before and after the adsorption experiments, it is necessary to have an idea of the hair structure. Hair is composed of distinct structures such as the cuticle, the cortex and the cell membrane complex. Cuticles are the most external part of the hair and contain an external layer of fatty acids connected to a protein layer, which is responsible for the hydrophobic character of hair. On the other hand, the cortex is the principal component of the hair and forms the matrix where other proteins such as keratin are located. The other part of the structure is the cell membrane complex which is responsible for the hair's natural moisture, brightness, transparency and hydration. Its outer lipid layer forms the epicuticle and the inner lipid layer is located between the cuticle cells. When hair is dyed, the dye molecules interact with the inner hair fibers. In this way, the structure of dyed hair is slightly different from that of natural hair, as the structure is not really affected by the dye treatment. $^{35}$

Therefore, the dyed hair treated in the experiments was further characterized using a scanning electron microscope (SEM).

Fig. 5 and 6 show the SEM images of the dyed hair surface corresponding to different treatments, at magnifications of 2000 and 10 000, respectively. They also show a control (Fig. 5a and 6a), where dyed hair was not subjected to the action of any surfactant or biosurfactant. Additionally, the figures contain images, corresponding to Fig. $5 \mathrm{~d}$ and $6 \mathrm{~d}$, of dyed hair that was treated with a cationic chemical surfactant, CTAB, under the same operational conditions $\left(50{ }^{\circ} \mathrm{C}, 2 \mathrm{~min}\right.$ and $\left.300 \mathrm{mg} \mathrm{L}^{-1}\right)$ as those established in experiment 11 (Table 2). These conditions were chosen after observing that BS was adsorbed in greater quantity onto dyed hair than in other experiments. Fig. 5b and $6 \mathrm{~b}$ correspond to experiment 7 (when almost no BS was adsorbed), whereas Fig. $5 c$ and $6 \mathrm{c}$ represent the hair surface morphology of experiment 11 (highest adsorption capacity of BS).

At a magnification of $2000 \times$, it can be seen that dyed hair treated with the biosurfactant (Fig. $5 \mathrm{~b}$ and c) has thicker cuticles with more homogeneous borders, and cuticles that lie flatter than those in untreated dyed hair samples (Fig. 5a) or samples treated with CTAB (Fig. 5d). This observation is corroborated by the pictures obtained at a magnification of $10000 \times$ (Fig. 6a-d), even in the samples where the biosurfactant concentrations adsorbed were negligible (Fig. 6b).

In regards to the treatment with CTAB (Fig. $5 \mathrm{~d}$ and $6 \mathrm{~d}$ ), the cuticle conveys a more damaged aspect, in comparison with that observed in Fig. $5 \mathrm{c}$ and $6 \mathrm{c}$ since it does not look as homogeneous as the others, and has apparent breaks in the surface. Regarding the use of synthetic surfactants, Wagner and collaborators $^{17}$ identified some damage, in terms of protein 
a)

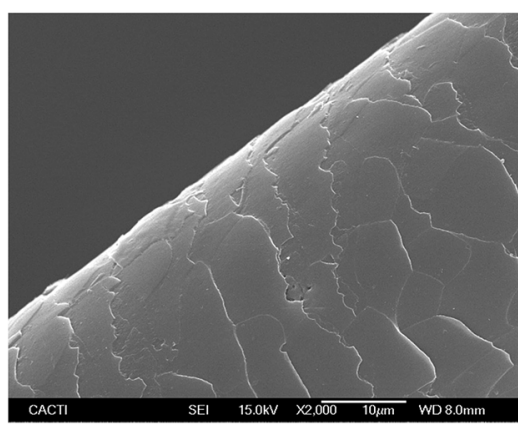

c)

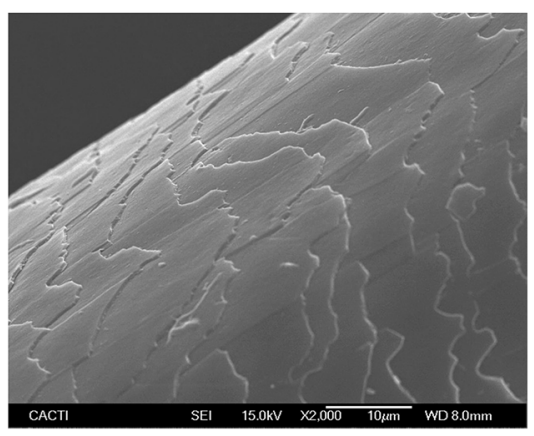

b)

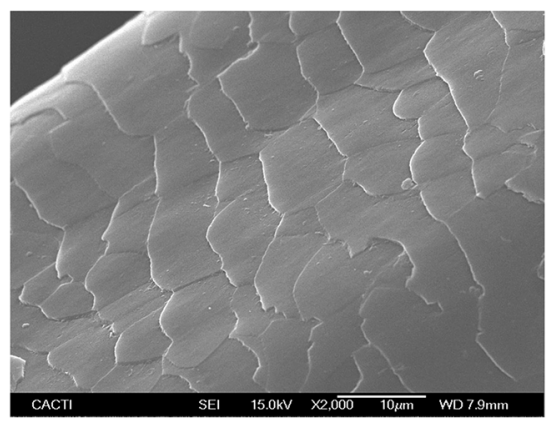

d)

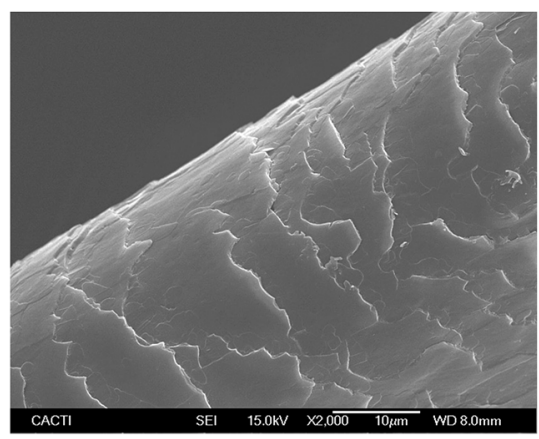

Fig. 5 SEM images corresponding to dyed hair at a magnification of 2000x: (a) untreated dyed hair (control), (b) dyed hair treated with the biosurfactant from CSL (lowest adsorption), (c) dyed hair treated with the biosurfactant from CSL (highest adsorption) and (d) dyed hair treated with CTAB, respectively.

a)

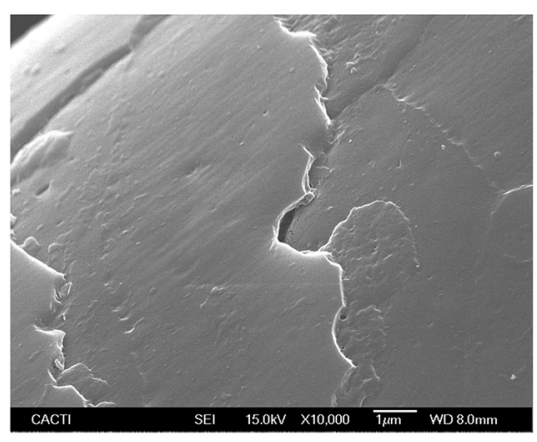

c)

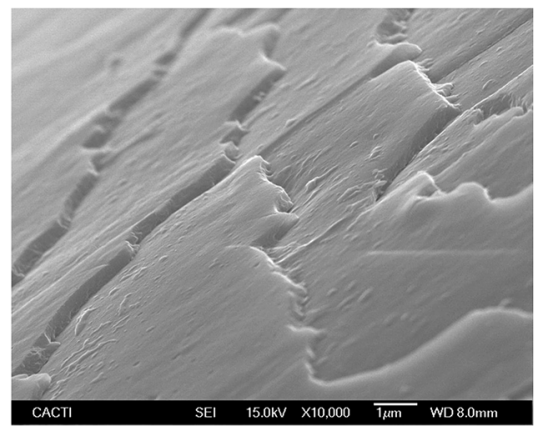

b)

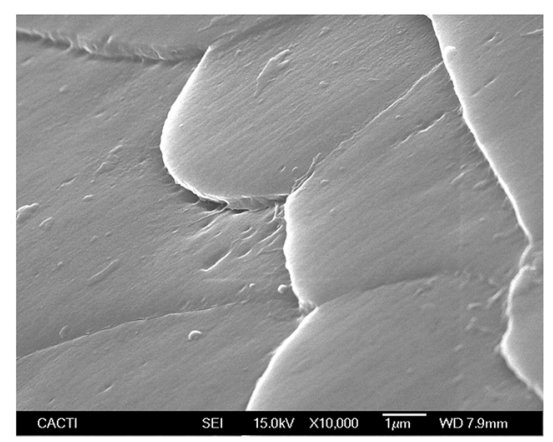

d)

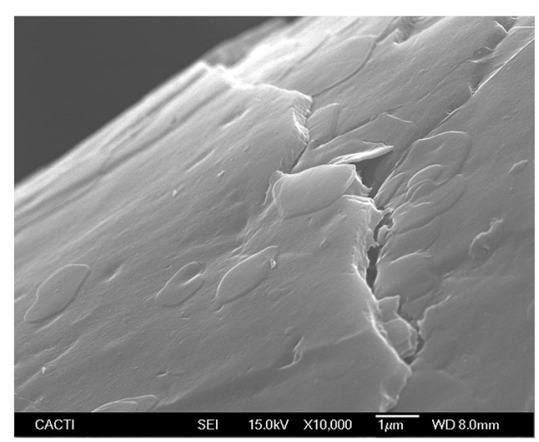

Fig. 6 SEM images corresponding to dyed hair at a magnification of $10000 \times$ : (a) untreated dyed hair (control), (b) dyed hair treated with the biosurfactant from CSL (lowest adsorption), (c) dyed hair treated with the biosurfactant from CSL (highest adsorption) and (d) dyed hair treated with CTAB, respectively. 
loss, when the hair was treated with sodium dodecyl sulfate (SDS), and showed SEM images, in which the hair cuticle presented some cracks and breaks over the surface, as well as angulated borders, which is in good agreement with the images obtained in the current work, thus corroborating the potential application of the biosurfactant extract, obtained from CSL, in dyed hair formulations.

\section{Conclusions}

Biosurfactant extract, obtained from corn steep liquor, exhibited good properties suggesting its ideal inclusion in the formulations of conditioners and mild shampoos, since the results showed the maximum adsorption capacity of biosurfactant onto dyed hair obtained up to now (10 $\left.549 \mu \mathrm{g} \mathrm{g}^{-1}\right)$. Moreover, it was corroborated that synthetic surfactants, such as CTAB, cause more damage to hair structure than the biosurfactant extracted from CSL, which maintains the hair structure in a good state and obtains more defined cuticles. Therefore, it would be interesting to study the inclusion of this biosurfactant in personal care and cosmetic formulations.

\section{Conflicts of interest}

The authors declare no competing financial interests.

\section{Acknowledgements}

This study was supported by the Spanish Ministry of Economy and Competitiveness (MINECO) under the project CTM201568904 (FEDER funds). X. Vecino is also grateful for her Juan de la Cierva contract (FJCI-2014-19732). L. Rodríguez-López acknowledges the Spanish Ministry of Education, Culture and Sport for her pre-doctoral fellowship (FPU15/00205).

\section{References}

1 X. Vecino, L. Barbosa-Pereira, R. Devesa-Rey, J. M. Cruz and A. B. Moldes, J. Agric. Food Chem., 2014, 62, 5451.

2 X. Vecino, L. Barbosa-Pereira, R. Devesa-Rey, J. M. Cruz and A. B. Moldes, Bioprocess Biosyst. Eng., 2015, 38, 1629.

3 L. Rodriguez-López, X. Vecino, L. Barbosa-Pereira, A. B. Moldes and J. M. Cruz, Food Funct., 2016, 7, 3724.

4 X. Vecino, J. M. Cruz, A. B. Moldes and L. R. Rodrigues, Crit. Rev. Biotechnol., 2017, DOI: 10.1080/07388551.2016.1269053.

5 C. C. Rossi, J. F. Santos-Gandelman, E. M. Barros, V. M. Alvarez, M. S. Laport and M. Giambiagi-deMarval, Lett. Appl. Microbiol., 2016, 63, 215.

6 M. A. D. De Rienzo, P. Stevenson, R. Marchant and I. M. Banat, FEMS Microbiol. Lett., 2016, 363, 224.

7 E. J. Gudiña, V. Rocha, J. A. Teixeira and L. R. Rodrigues, Lett. Appl. Microbiol., 2010, 50, 419.

8 J. M. Luna, R. D. Rufino, L. A. Sarubbo, L. R. Rodrigues, J. A. Teixeira and G. M. de Campos-Takaki, Curr. Microbiol., 2011, 62, 1527.

9 P. Chiewpattanakul, S. Phonnok, A. Durand, E. Marie and B. W. Thanomsub, J. Microbiol. Biotechnol., 2010, 20, 1664.
10 C. Duarte, E. J. Gudiña, C. F. Lima and L. R. Rodrigues, $A M B$ Express, 2014, 4, 1.

11 E. Gharaei-Fathabad, Am. J. Drug Discovery Dev., 2011, 1, 58.

12 L. R. Rodrigues and J. A. Teixeira, Biosurfactants, Springer, New York, 2010, pp. 75-87.

13 E. J. Gudiña, V. Rangarajan, R. Sen and L. R. Rodrigues, Trends Pharmacol. Sci., 2013, 34, 667.

14 M. Elshikh, R. Marchant and I. M. Banat, FEMS Microbiol., 2016, 363, 213.

15 L. Rodrigues, I. M. Banat, J. Teixeira and R. Oliveira, J. Antimicrob. Chemother., 2006, 57, 609.

16 G. Ran, Y. Zhang, Q. Song, Y. Wang and D. Cao, Colloids Surf., B, 2009, 68, 106.

17 R. D. C. C. Wagner and I. Joekes, Colloids Surf., B, 2005, 41, 7.

18 M. Rincón-Fontán, L. Rodríguez-López, X. Vecino, J. M. Cruz and A. B. Moldes, RSC Adv., 2016, 6, 63064.

19 T. Morita, M. Kitagawa, S. Yamamoto, A. Sogabe, T. Imura, T. Fukuoka and D. Kitamoto, J. Oleo Sci., 2010, 59, 267.

20 N. Kosaric and F. V. Sukan, Biosurfactants: Production and Utilization-Processes, Technologies, and Economics, CRC Press, 2014, vol. 159.

21 C. A. B. De Gusmao, R. D. Rufino and L. A. Sarubbo, World J. Microbiol. Biotechnol., 2010, 26, 1683.

22 S. Mukherjee, P. Das and R. Sen, Trends Biotechnol., 2006, 24, 509.

23 A. B. Moldes, A. M. Torrado, M. T. Barral and J. M. Domínguez, J. Agric. Food Chem., 2007, 55, 4481.

24 O. M. P. Rivera, A. B. Moldes, A. M. Torrado and J. M. Domínguez, Process Biochem., 2007, 42, 1010.

25 O. M. Portilla, B. Rivas, A. Torrado, A. B. Moldes and J. M. Domínguez, J. Sci. Food Agric., 2008, 88, 2298.

26 S. Cortés-Camargo, N. Pérez-Rodríguez, R. P. D. S. Oliveira, B. E. B. Huerta and J. M. Domínguez, Ind. Crops Prod., 2016, 79, 258.

27 F. Mariotti, D. Tomé and P. Mirand, Crit. Rev. Food Sci. Nutr., 2008, 48, 177.

28 N. Vanittanakom, W. Loeffler, U. Koch and G. Jung, J. Antibiot., 1986, 39, 888.

29 J. Kijlstra, J. S. Namnath, R. Pontzen, U. WachendorffNeumann and C. Waterman, Compositions and methods for controlling fungal and bacterial diseases in plants, U.S. Patent Application No. 14/724,698, 2015.

30 N. P. Price, A. P. Rooney, J. L. Swezey, E. Perry and F. M. Cohan, FEMS Microbiol. Lett., 2007, 271, 83.

31 L. Rodriguez-López, M. Rincón-Fontán, X. Vecino, A. B. Moldes and J. M. Cruz, J. Surfactants Deterg., 2017, 20, 207.

32 Handbook of detergents, part E: applications, ed. U. Zoller, CRC Press, 2008, vol. 141.

33 M. K. Polano, J. Soc. Cosmet. Chem., 1968, 19, 3.

34 G. Imokawa, K. Sumura and M. Katsumi, J. Am. Oil Chem. Soc., 1975, 52, 484.

35 S. Aparecida da França, M. Ferrera Dario, V. Brigatto Esteves, A. Rolim Baby and M. V. Robles Velasco, Cosmetics, 2015, 2, 110. 